\title{
ATTENUATED TOTAL REFLECTANCE - FOURIER TRANSFORM INFRARED SPECTROSCOPY APPLIED FOR THE EVALUATION OF ESSENTIAL OILS' PATTERN RECOGNITION AND THERMO-OXIDATIVE STABILITY: A COMPARATIVE STUDY
}

\author{
RAMONA MARIA POPA ${ }^{a}$, FLORINELA FETEA ${ }^{a}$, CARMEN SOCACIU $^{a, b,{ }^{*}}$
}

\begin{abstract}
The Attenuated Total Reflectance-Fourier Transform Infrared Spectroscopy was applied for the evaluation of specific patterns for six different essential oils obtained from aromatic plants (thyme, oregano, tea tree) or common spices (clove, cinnamon, juniper). The spectral fingerprints were recorded using a Shimadzu IR Prestige-21 spectrometer in the middle infrared region (3500-650 $\mathrm{cm}^{-1}$ ) before and after thermal oxidation in hot air. In parallel, the peroxide index, cis-trans isomerization and lipoperoxidation were evaluated to determine their oxidative stability. Each oil showed specific FTIR spectra, especially in the fingerprint region $\left(1650-650 \mathrm{~cm}^{-1}\right)$, in good agreement with our previous GC-MS analysis of the same samples. The spectral patterns in this region were also correlated with their oxidative stability, chemically determined. The spectral data were processed by multivariate analysis (Metaboanalyst 5.0 software) and the discriminatory analysis helped to identify each oil pattern.

This study combined the capability of ATR-FTIR-MIR spectroscopy to realize a pattern recognition of essential oils as a rapid and non-destructive method, as well the oxidative modifications, easier than chemical routine tests. This study contributes to the control of quality, authenticity, and safety of essential oils and can be extended to other extracts, used as additives or ingredients in food or cosmetic products.
\end{abstract}

Keywords: essential oils fingerprinting, Attenuated Total Reflectance Mid Infrared Spectroscopy, thermal oxidation

a Department of Food Science, Faculty of Food Science and Technology, University of Agricultural Sciences and Veterinary Medicine Cluj-Napoca, Cluj-Napoca RO-400372, Romania

b Research Center for Applied Biotechnology, Proplanta, Cluj-Napoca, RO-400478 Romania Corresponding author: carmen.socaciu@usamvcluj.ro 


\section{INTRODUCTION}

The Fourier Transform Infrared Spectroscopy (FTIR) is a nondestructive, fast, reproducible analytical method useful to fingerprint different food products. It can identify specific vibration bands, characteristic to functional groups of different molecules [1-3]. The Attenuated Total Reflectance-Fourier Transform Spectroscopy (ATR-FTIR) is the most suitable technique to highlight the specific imprint of liquid samples, including plant oils and volatile essential oils (EOs), to check their authenticity, traceability and adulteration $[2,4-6]$. Moreover, it is useful to identify oxidative processes that modify the functional groups (alcohols, aldehydes, ketones, epoxides, peroxides, acids). The ATR-FTIR spectral data are usually collected in the whole middle Infrared region (MIR), from 4000 to $400 \mathrm{~cm}^{-1}$ including the fingerprint region $1800-650 \mathrm{~cm}^{-1}$.

It is also an excellent tool for quantitative analysis, because the intensities of the spectral bands are proportional to concentration. It helps to distinguish oils from different botanical origins using specific wavenumber domains coupled with non-supervised classification techniques [7-9].

Different reviews and experimental data have been published, about the FTIR spectroscopy applied for the characterization of edible oils, and to compare it with other physical and chemical properties, e.g. lodine value peroxide and saponification index [6, 10-12], as well as for the freshness evaluation and detection of adulteration [13]. A large majority of data refer to olive oil adulteration with low-cost edible oils $[12,14]$ or to assess oil oxidation [15-17]. Moreover, the determination of edible oil authenticity, compared with the more accurate but time-consuming, laborious, and expensive chromatography-based methods (GC-MS) is advantageous being a more rapid and cheaper solution. Considering the advantages of being non-destructive, cost effective and environmentally friendly, using small amounts of liquid samples and simple preparation procedures $[5,18]$. Meanwhile FTIR spectroscopy was found to be superior for the discrimination and classification of edible oil comparing to FT-Raman and FT-NIR [19, 20].

The applicability of FTIR-MIR spectroscopy in assessing oils fingerprinting quality \& safety parameters is crucially dependent on the chemometric methods, including calibrations with large and representative samples $[13,21]$. The common chemometric classification includes nonsupervised approaches e.g. principal component analysis (PCA), cluster analysis (CA), while the supervised methods include partial least squares with discriminant Analysis (PLSDA), linear discriminating analysis (LDA) and principal component regression (PCR) [22]. The Random Forest (RF) algorithm is applied as a superior classifier useful for food adulteration, for 
classification and authentication in chemometrics [23]. The web-based software MetaboAnalyst 5.0 (www.metaboanalyst.ca/) is a new integrated tool which accepts a variety of input data (from NMR and MS to FTIR peak lists) and offers several chemometric options for data processing, from univariate (ANOVA t-test) to multivariate statistical analysis, PCA, PLS-DA, $\mathrm{CA}$, heatmaps, RF and more sophisticated statistical or machine learning methods [11].

EOs include a variety of volatile molecules such as terpenes and terpenoids (esters, aldehydes, acids, alcohols, ketones, and ethers derived from terpenes), phenol-derived aromatic and aliphatic components [24-27]. EOs proved to have multiple biological actions, such as antioxidant, bactericidal, virucidal, fungicidal, antiparasitic, insecticidal effects [28-30] being used in food industries, as natural flavors or in different pharmaceutical and cosmetic formulations. Therefore, the quality, authenticity and safety of EOs is of high scientific interest and involves high resolution analytical procedures, e.g. gas-chromatography coupled with mass spectrometry, infrared spectroscopy, magnetic resonance [4, 31, 32].

EOs are extracted from aromatic herbs and spices, vegetables and fruits, many of them being described in EMA monographies (https://www.ema.europa.eu/en/medicines/herbal) and in European pharmacopeia [33]. The ATR-FTIR-MIR method can be adapted for a global quantification of EOs free or dissolved in edible oils or encapsulated in hydrocolloids or powders and can be used also to compare their chemical profile during storage, as recently reported $[34,35]$. This technique allows a short analysis time, minimum sample preparation [36, 37].

Recently, the characterization of the volatile fractions of EOs of some Lamiaceae spices in relation to their activities was reported [38, 39]. Thyme (Thymus vulgaris $L$.) includes different subspecies and varieties and is used frequently as a spice or medicinal remedy with anti-inflammatory and antibacterial effects, its oily extracts being rich in thymol and terpinene-4-ol $[26,40,41]$ beside flavonoids and triterpenoids. Oregano (Origanum vulgare $L$.) is rich in monoterpenoids (thymol and carvacrol) and sesquiterpenoids with antiinflamatory, antispasmodic, antioxidant, antifungal and antibacterial activity $[42,43]$. Juniperus (Juniperus communis $L$.) is a coniferous tree, its oil contains monoterpenes (approx. 58\%), mainly $\alpha$-pinene, $\beta$-myrcene and sabinene [44]. The industrial applications in relation to the chemical composition and antimicrobial activity obtained from its berries was recently confirmed [45]. Tea tree (Melaleuca sp.) is used for its essential oil, the main oil components are monoterpenes and sesquiterpenes, and their oxygenated analogues $[46,47]$ with antiseptic, cytotoxic, antibiotic, anti-inflammatory and antioxidant activity. Clove (Syzygium aromaticum L.) is a rich source of eugenol, 
eugenol acetate, gallic acid, having a high potential for pharmaceutical, cosmetic and food applications due to its antioxidant and antimicrobial effect $[48,49]$. Cinnamon (Cinnamomi Cortex) oil is extracted from its bark and contains mainly cinnamaldehyde (up to $87,23 \%$ ) coumarin, cinnamic alcohol and acid, its biological activity (antioxidant, antidiabetic, thrombocyte antiaggregation, antifungal and antibacterial) as recently described [50-53]. We applied previously the GC-MS analysis for a comparative identification of similar six EOs as well the behavior of EOs in different formulations [54, 55].

This study aims to apply the ATR-FTIR-MIR spectroscopy for the evaluation of the specific pattern of six types of essential oils obtained from aromatic plants (thyme, oregano, tea tree) or common spices (clove, cinnamon, juniper). The specific spectral fingerprints were recorded and the oxidative stability after an induced thermal oxidation in hot air was investigated. All data were processed by statistical multivariate analysis using the online Metaboanalyst 5.0 software and the specific patterns of their recognition, authenticity, and stability were established.

\section{RESULTS AND DISCUSSION}

\section{Physical characteristics}

Some physical characteristics of the studied EOs are presented in Table 1.

Table 1. Comparative physical characteristics of the 6 essential oils: density, refractive index, rotational power.

\begin{tabular}{|c|c|c|c|}
\hline Essential oil & Relative Density & Refractive index & Rotational power \\
\hline Thyme & 0.9220 & 1.4980 & -2.7 \\
\hline Oregano & 0.9500 & 1.5120 & +1.1 \\
\hline Juniper & 0.8610 & 1.4770 & -6.2 \\
\hline Cloves & 1.0580 & 1.5320 & -1.6 \\
\hline Cinnamon & 1.0200 & 1.5860 & -1.1 \\
\hline Tea tree & 0.9020 & 1.4770 & +10.2 \\
\hline
\end{tabular}

The highest refractive index was seen for Cinnamon, Clove and Oregano oils, in parallel with highest density for these oils. The rotational power was very different, from +10.2 to -6.2 and was dependent on the sums of the different optical active terpenoids found in these essential oils. Tea tree had a high dextrogir rotational power which may be explained by its rich composition in (+)terpiene-4-ol, while Juniper the highset levogir power due to (-) alpha pinene. 
ATTENUATED TOTAL REFLECTANCE - FOURIER TRANSFORM INFRARED SPECTROSCOPY ...

\section{ATR-FTIR-MIR spectra}

The Infrared absorption bands observed before and after the induced thermal oxidation are characteristic to different types of bonds and functional groups found in EOs, considering two MIR domains, $1800-650 \mathrm{~cm}^{-1}, 3600-$ $2500 \mathrm{~cm}^{-1}$ ), as presented in Figure $1 \mathrm{~A}-\mathrm{G}$.

For comparison, the same spectra were recorded, as a control, for Sunflower oil, before and after the induced thermal oxidation.

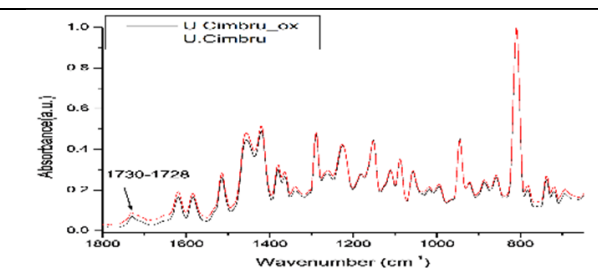

A1.

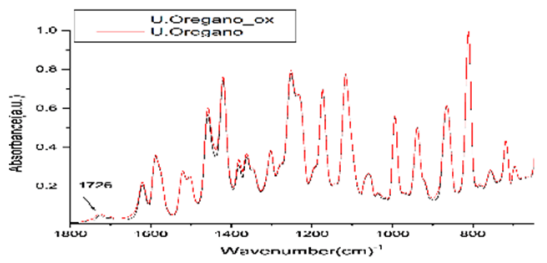

B1.

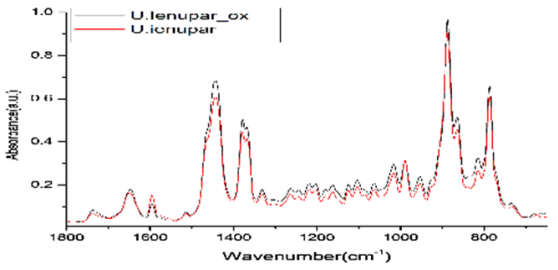

C1.

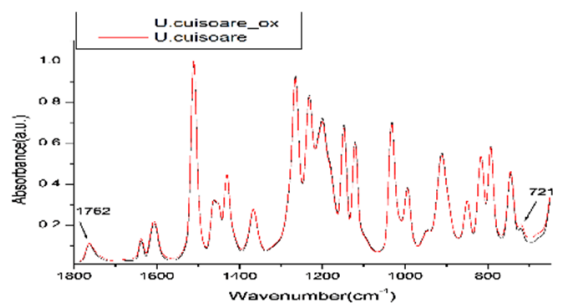

D1.

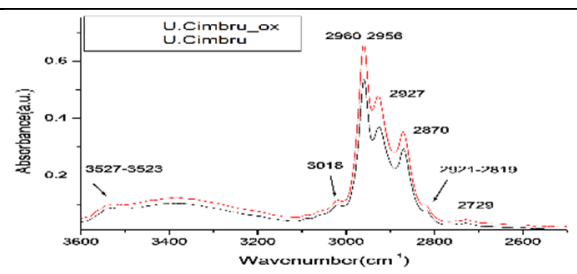

A2.

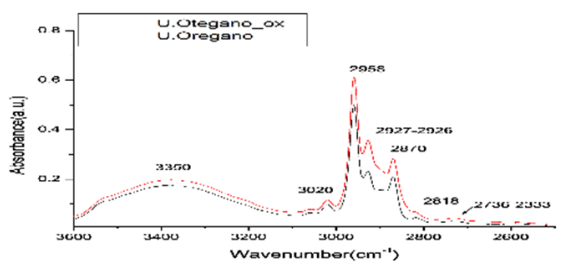

B2.

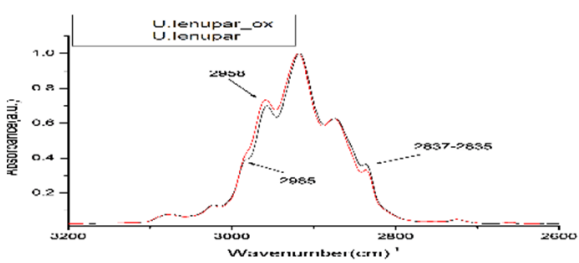

C2.

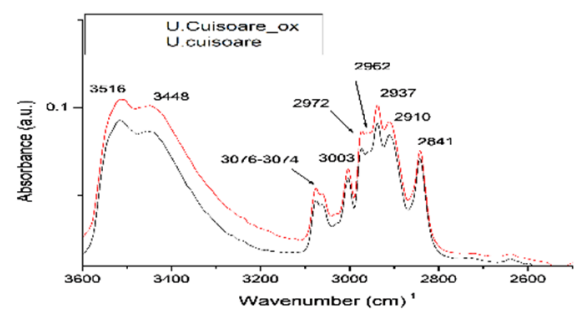

D2. 


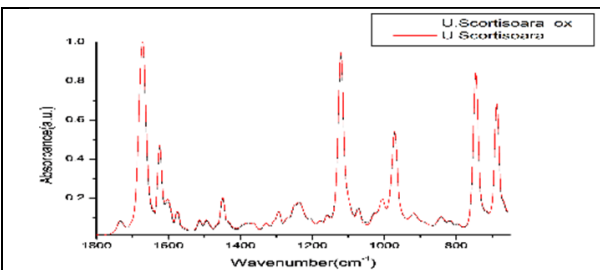

E1.

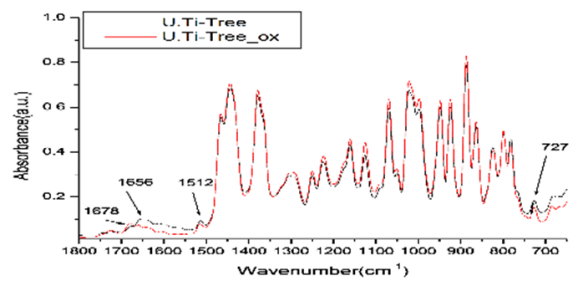

F1.

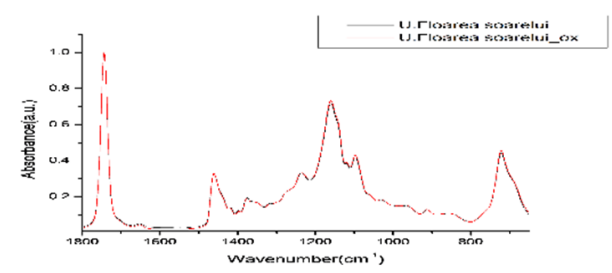

G1.

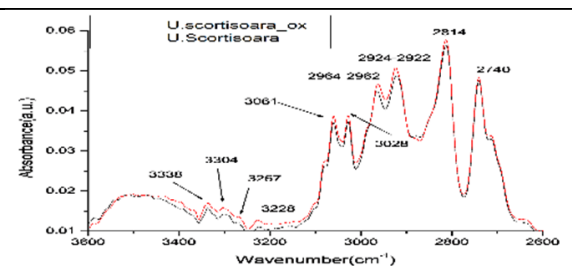

E2.

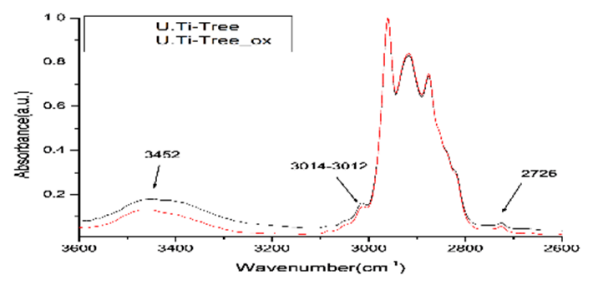

F2.

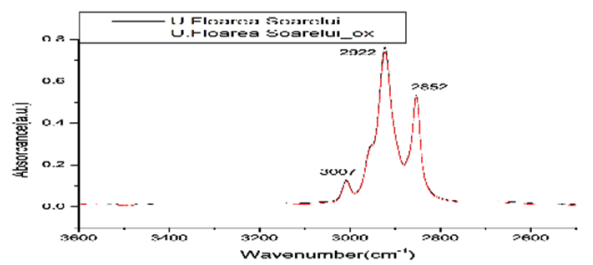

G2.

Figure 1. FTIR spectra in the region $1800-650 \mathrm{~cm}^{-1}$ and $3600-2500 \mathrm{~cm}^{-1}$ of six essential oils, before (black) and after induced thermal oxidation (red).

A1-A2. Thyme (Cimbru). B1-B2. Oregano. C1-C2. Juniperus (lenupăr).

D1-D2. Clove (Cuişoare). E1-E2. Cinnamon (Scorţişoară). F1-F2. Tee tree (Ti-Tree). G1-G2. Sunflower oil (Floarea Soarelui) as control.

All spectra included peaks in four zones, disregarding the zones specific to nitrogen compounds: $1\left(1000-650 \mathrm{~cm}^{-1}\right), 2\left(1470-1000 \mathrm{~cm}^{-1}\right), 3$ $\left(1750-1500 \mathrm{~cm}^{-1}\right)$ and $4\left(3600-2700 \mathrm{~cm}^{-1}\right)$, attributed to different stretching or deformation vibrations known to characterize oils (Table 4).

The spectral fingerprints for thyme oil (Figure 1A1-A2) show dominant signals with characteristic frequencies in zones 1-2 and 4, where the signal intensities were higher than 0.38 . Such signals are good markers to fingerprint thyme: 810vs, 945m, 1551m, 1226m, 1419m, 1456m, 2927m, 2960s, 3527m (Table 2). No specific signals (fi, i, $\mathrm{m}$ ) were identified in zone 3 . Oxidation reduced some intensities only in zone 4 , indicating small changes in aliphatic chain vibrations $\mathrm{CH}_{2}$ and $\mathrm{CH}_{3}$.

For oregano (Figure 1B1-B2), the fingerprints include specific wavenumbers and intensities at $717 \mathrm{~m}, 866 \mathrm{~s}, 939 \mathrm{~m}, 993 \mathrm{~m}, 1116 \mathrm{~s}, 1172 \mathrm{~s}, 1232 \mathrm{~s}$, 
1251s, 1419s, 1458s, 2958s, 3350m. Oxidation reduced some intensities, but not significantly, indicating similar changes in vibrations in aliphatic chains.

For juniper (Figure 1C1-C2), the specific frequencies in zones 1-2 and 4 , with signal intensities higher than 0.38 were identified. The specific pattern is different, include peaks at $786 \mathrm{~s}, 864 \mathrm{~m}, 889 \mathrm{vs}, 1367 \mathrm{~m}, 1379 \mathrm{~m}, 1442 \mathrm{~s}$, 2875 s, 2918vs, 2985s. To notice in this case no peaks were visible at wavenumbers higher than $2985 \mathrm{~cm}^{-1}$.

The spectral fingerprints of clove oil (Figure 1D1-D2) look different from thyme, oregano or juniper, some signals are dominant in both samples (clove oil and oxidized clove oil). These signals represent specific imprints of clove: $744 \mathrm{~m}, 792 \mathrm{~m}, 817 \mathrm{~m}, 912 \mathrm{~m}, 1120 \mathrm{~m}, 1147 \mathrm{~s}, 1232 \mathrm{~s}, 1265 \mathrm{vs}, 1431 \mathrm{~m}$, $1510 v s, 3448$ s and $3515 \mathrm{~s}$. Oxidation reduced some intensities, but only in zone $4\left(2700-3600 \mathrm{~cm}^{-1}\right)$.

Cinnamon pattern (Figure 1E1-E2) is highly specific and different, with peaks at $686 \mathrm{~s}, 746 \mathrm{~s}, 972 \mathrm{~m}, 1120 \mathrm{vs}, 1624 \mathrm{~m}, 1672 \mathrm{vs}, 3338$ and $3304 \mathrm{~m}$. Oxidation did not reduce intensities, insignificantly even in zone 4 . The peak at $686 \mathrm{~cm}^{-1}$ corresponds to the vibration specific to alkenes, while peaks at $1672,1624 \mathrm{~cm}^{-1}$ and $1734 \mathrm{~cm}^{-1}$ correspond to the C-O carbonyl bond from aldehydes vibration, indicators of cinnamic aldehyde, a marker of cinnamon.

Tea tree (Figure 1F1-F2) had also specific fingerprint, with multiple peaks, at $781 \mathrm{~m}, 798 \mathrm{~m}, 823 \mathrm{~m}, 864 \mathrm{~m}, 887 \mathrm{~s}, 923 \mathrm{~s}, 948 \mathrm{~s}, 997 \mathrm{~m}, 1022 \mathrm{~s}, 1068 \mathrm{~m}$, $1126 \mathrm{~m}, 1161 \mathrm{~m}, 1379 \mathrm{~s}, 1442 \mathrm{~s}, 1465 \mathrm{~m}, 2875 \mathrm{~s}, 2916 \mathrm{~s}, 3452 \mathrm{~m}$ different from the other oils. Oxidation does not affect the pattern, only slight increases of the signals above $3014 \mathrm{~cm}^{-1}$.

The sunflower oil imprint (Figure 1G1-G2) is the poorest in signals, some dominant signals can be seen at $721 \mathrm{~m}, 1097 \mathrm{~m}, 1120 \mathrm{~m}, 1159 \mathrm{~s}, 1743 \mathrm{vs}$, $2852 \mathrm{~m}, 2922 \mathrm{~s}, 3007 \mathrm{~m}$ and no signals at higher wavenumbers. No changes after induced oxidation are noted, since sunflower oil contains lipophilic antioxidants, mainly tocopherols and carotenoids which act as antioxidants.

To conclude, the absorption frequencies in the ATR-FTIR-MIR spectra in the fingerprint region $\left(1800-650 \mathrm{~cm}^{-1}\right)$ were assigned to be specific to the different EOs: absorptions characteristic to the aromatic ring between 890-730 $\mathrm{cm}^{-1}(1)$, attributed to aliphatic long chain hydrocarbons at aprox. $720 \mathrm{~cm}^{-1}(2)$ and absorptions in the region $1750-1500 \mathrm{~cm}^{-1}$ attributed to carbonyl compounds at approx. $1750 \mathrm{~cm}^{-1}$ and to $\mathrm{C}=\mathrm{C}$ absorption at $1600 \mathrm{~cm}^{-1}(3)$. In the region $3600-2700 \mathrm{~cm}^{-1}$ three spectral areas showed visible changes for all EOs: absorptions in the zone $2920-2850 \mathrm{~cm}^{-1}$ corresponding to stretching vibration in the $\mathrm{CH}_{2}$ aliphatic chain (1), in the range $3003-3007 \mathrm{~cm}^{-1}$ corresponding to stretching vibration of the double bond cis specific to unsaturated fatty acids, the decreased intensity being a marker of cis-trans isomerization (2) and the band at $3473 \mathrm{~cm}^{-1}$ attributed to the $\mathrm{O}-\mathrm{H}$ stretching vibration of hydroperoxides (3).

The comparative image of the patterns of different EOs and their specific FTIR markers are summarized in Table 2. 
Table 2. Wavenumbers considered as markers for EOs pattern recognition*.

\begin{tabular}{|l|c|c|c|c|}
\hline Nr. & EO & $\begin{array}{c}\text { Very strong } \\
\text { signals (vs) }\end{array}$ & Strong signals (s) & Medium signals $(\mathrm{m})$ \\
\hline 1. & Thyme & 810 & 2960 & $\begin{array}{c}945,1551,1226,1419, \\
1456,2927,3018\end{array}$ \\
\hline 2. & Juniperus & 889,2918 & $786,1442,2875,2958,3022$ & $864,1367,1379$ \\
\hline 3. & Oregano & - & $\begin{array}{c}866,1116,1172,1232, \\
1251,1419,1458,2958,3020\end{array}$ & $717,939,993$ \\
\hline 4. & Cloves & 1265,1510 & 1147,1232 & $744,792,817,912,1120,1431$ \\
\hline 5. & Cinnamon & 1120,1672 & 686,746 & $972,162,3061,3308$ \\
\hline 6. & Tea tree & - & $887,923,948,1022,1379$, & $781,798,823,864,997$, \\
$1442,2875,2916$ & $1068,1126,1161,1465$ \\
\hline 7. & Sunflower & 1743 & $1159,2922,3007$ & $721,1097,1120,2852$ \\
\hline
\end{tabular}

*The abbreviations for intensities are marked in parentheses (vs =>0.9; $s=>0.6 ; m=>0.38$ ).

\section{Chemometrics: Multivariate analysis for EOs pattern recognition}

The PLSDA Analysis was first applied to identify the discrimination between samples from the two groups of EOs, a first one considered to belong to aromatic or medicinal plants MP (Thyme, Oregano and Tea tree) and the second one, the spice-group $S$ (Juniperus, Clove and Cinnamon). Figure $2 \mathrm{~A}$ and $\mathrm{B}$ represents the score plot of PLSDA and the VIP scores derived from loadings, which identify the relevant features (IR ranges) that may explain the discrimination between groups MP and S.

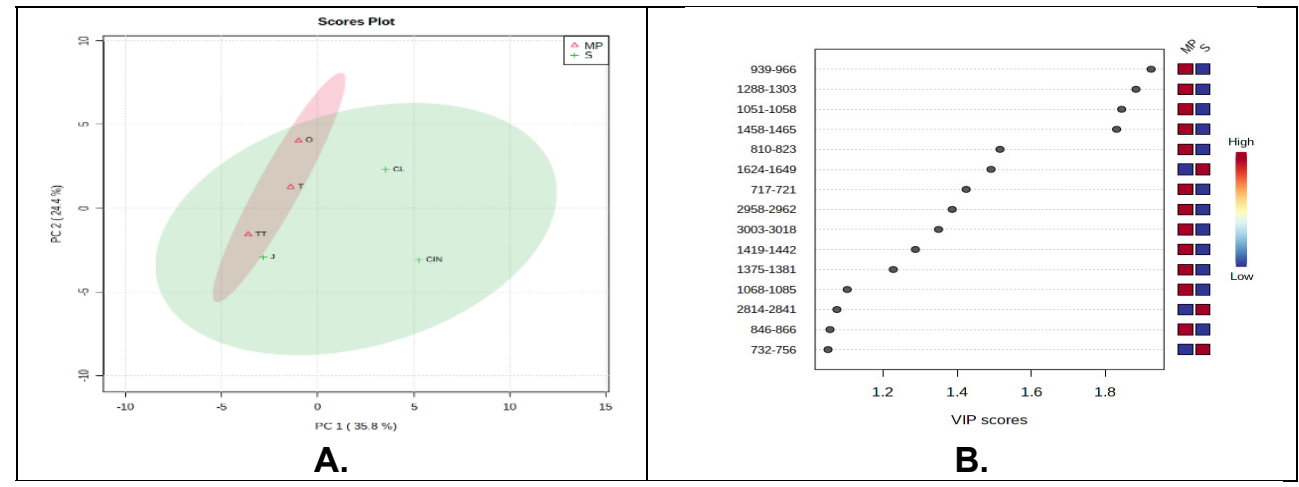

Figure 2. A. The PLSDA Score plot for samples' discrimination. B. VIP scores corresponding to wavenumbers (WN) which discriminate between groups MP and

S. T-thymus; O-oregano; TT-Tea tree; J-Jenuper; CIN-Cinnamon; CL-clove. 
As can be seen in Figure 1A, the co-variance of components PC1PC2 was $60 \%$, indicating an acceptable significance of discrimination. There are observed high similarities between $\mathrm{T}, \mathrm{O}$ and TT, close to J. CL and CIN show distinct patterns. The WN ranges which may be considered most relevant for this discrimination are 939-966, 1288-1303, 1051-1058, 1458$1465 \mathrm{~cm}^{-1}$. Applying the RF analysis (Mean Decrease Accuracy), the first 15 wavenumbers to be considered potential biomarkers for the discrimination of MP vs S groups are shown in Figure $3 \mathrm{~A}$.

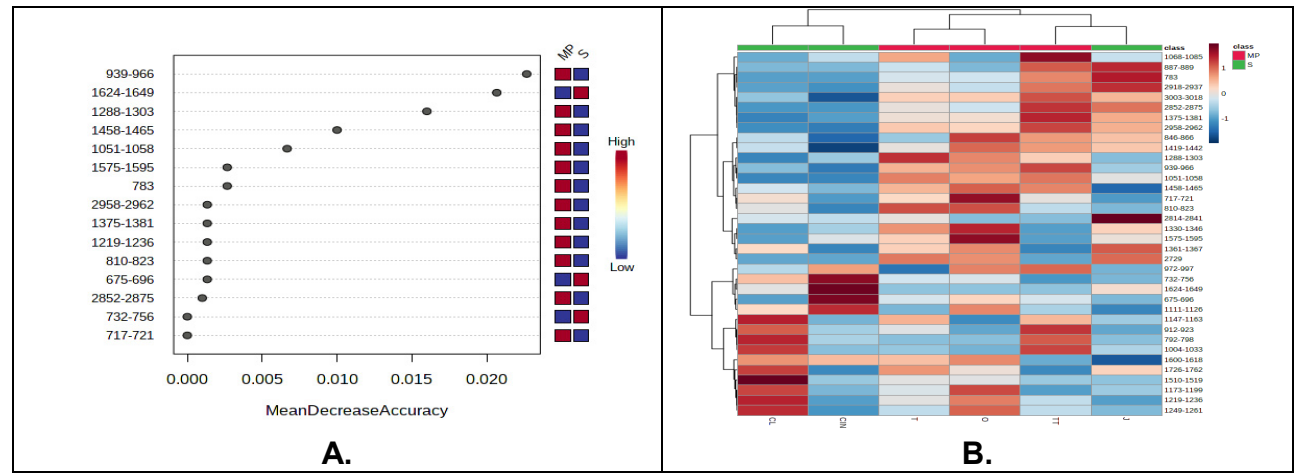

Figure 3. A. The MDA plot showing the wavenumbers to be considered biomarkers of discrimination between groups MP and S. B. The Heatmap showing above the sample clusters MP and S (red vs green) and the correlations between individual samples and FTIR intensities at different wavenumbers. T-thymus; O-oregano; TTTea tree; J-Jenuper; CIN-Cinnamon; CL-clove.

These are 939-966, 1624-1649, 1288-1303, 1458-1465 $\mathrm{cm}^{-1}$, very similar to the previous analysis (Figure 2B.). The intuitive image of correlations between EOs samples and the WN ranges are presented in heatmap (Figure 3B). One can see distinct patterns of each EO and the ranges which can be considered as authenticity fingerprint, as well the sample clusters MP and S (red vs green).

\section{Multivariate analysis to identify EOs stability against oxidation}

Based on the spectral fingerprints and the matrices representing the peak wavenumbers vs intensity, for each EOs sample, before and after induced thermal oxidation, the PLSDA and RF analysis showed the differences between the initial $(G)$ and final stage $(O x)$. Figure $4 A$ and $B$ represents the score plot of PLSDA and the VIP scores which identified the features (wavenumbers) that may explain the discrimination between sample groups $\mathrm{G}$ and $\mathrm{Ox}$. 


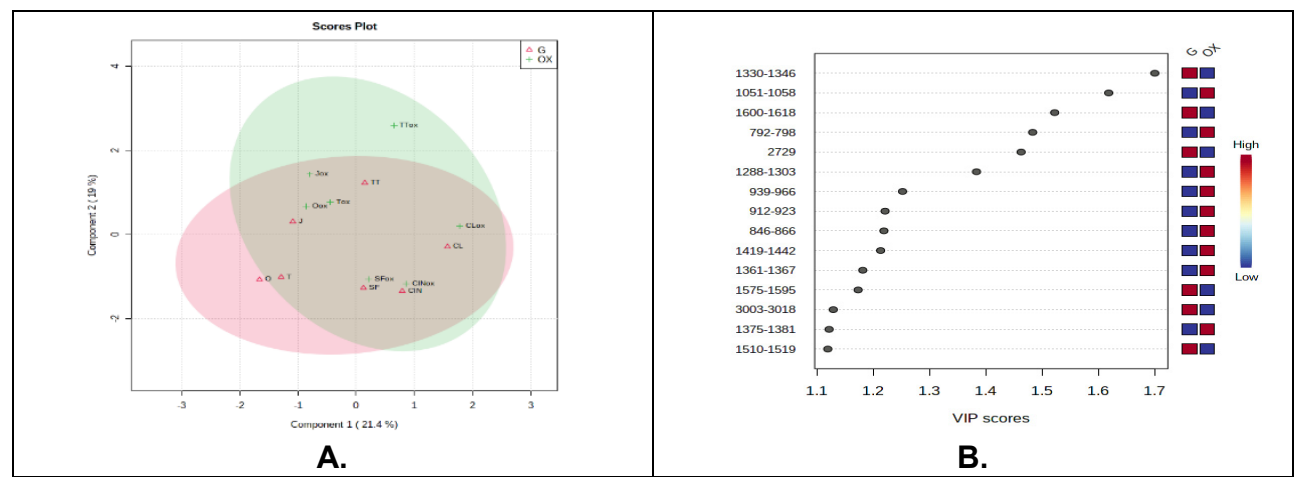

Figure 4.A. The PLSDA Score plot for the discrimination between EOs, at initial (G) and final stage (Ox). B. VIP scores corresponding to wavenumbers which discriminate between groups $\mathrm{G}$ and Ox. T-thymus; O-oregano; TT-Tea tree; J-juniper; CIN-Cinnamon; CL-clove.

As can be seen in Figure $4 \mathrm{~A}$, the co-variance of components PC1PC2 was $40.4 \%$, indicating weak discriminations between samples in initial and oxidized form. This indicates a good stability of samples against induced thermal oxidation, especially visible for SF, CIN and CL. The most labile seems to be $\mathrm{T}$ and $\mathrm{O}$ as indicated by the distance between the two stages in the plot. The WN ranges which may be considered most relevant for this discrimination are 1330-1346, 1051-1058, 1600-1618, 792-798, 1288-1303 $\mathrm{cm}^{-1}$ with higher VIP scores (Figure 4B). Applying the RF analysis and considering Mean Decrease Accuracy (MDA), the first 15 wavenumbers to be considered potential biomarkers for the discrimination of $\mathrm{G}$ vs ox groups are shown in Figure $5 \mathrm{~A}$.

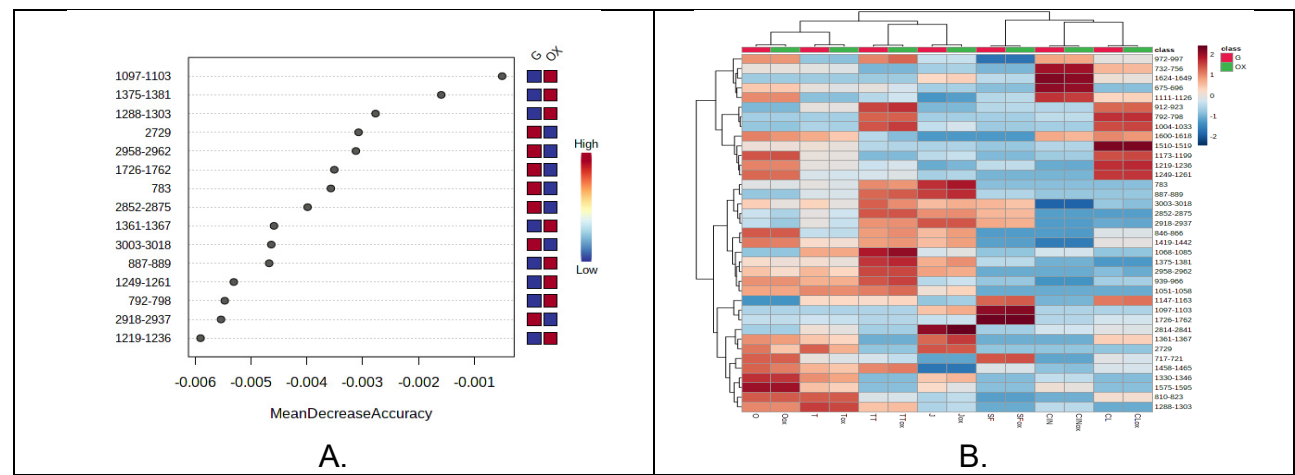

Figure 5.A. The MDA plot showing the wavenumbers to be considered biomarkers of discrimination between groups $\mathrm{G}$ and $\mathrm{Ox}$. B. The Heatmap showing the sample clusters and their correlations with the FTIR intensities at different wavenumbers, before $(G)$ and after thermal oxidation $(O x)$. 
These are 1097-1103, 1375-1381, 1288-1303, 2729, 2958-2962, 1726-1762, 1458-1465 $\mathrm{cm}^{-1}$, many being similar to the VIP score ranking (Figure 4B.). Here also, an intuitive image of correlations between EOs samples (at initial stage comparative to the final stage after induced oxidation) and the WN ranges are presented in heatmap (Figure 5B). One can see also the patterns of each $\mathrm{EO}$ and as well the similarities between the initial and oxidized forms (red vs green).

\section{Oxidative stability and cis-trans isomerization by induced thermal oxidation}

In parallel to FTIR spectroscopy, the usual parameters used for the identification of oxidation were determined, e.g., Peroxide Index (IP) and Lipid peroxidation by MDA test. Figure 6 shows the correlation plot between these parameters and the wavenumbers which were identified above, as relevant for the ATR-FTIR-MID spectral modifications. For these correlations there were considered the ratios between the oxidized/initial stage (OX/I), for each of the six EOs.

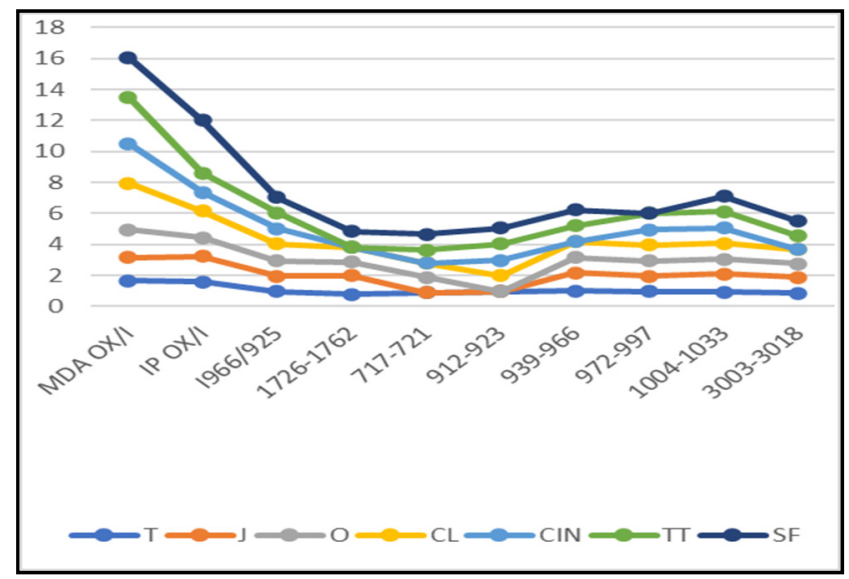

Figure 6. Correlations (expressed as ratios between the oxidized/initial stage) between the different parameters to characterize oxidation, from MDA-lipid peroxidation, to peroxide index (IP) and peak intensities of different FTIR ranges for the six EOs: Thyme(T), Juniper(J), Oregano(O), Clove (C), Cinnamon (CIN), Tea tree (TT), Sunflower (SF).

Also, considering that genuine plant-derived oils contain cis isomers of different unsaturated molecules, in agreement with FTIR application notes [56], the increase of the peak intensity at $966 \mathrm{~cm}^{-1}$ and of the intensity ratios 
$966 / 925 \mathrm{~cm}^{-1}$ can indicate the isomerization of cis trans isomers. It is known that trans fats are considered by-products of the thermal processing. Therefore, we considered these ratios to compare the behaviors of different EOs after induced thermal oxidation, as shown in table 3.

Table 3. Signal intensity ratios $966 / 925 \mathrm{~cm}^{-1}$, as indicators of cis-trans isomerizations, before (I) and after induced thermal oxidation.

\begin{tabular}{|c|c|}
\hline Essential oils & $\mathbf{I 9 6 6 / 9 2 5}$ Ox/I \\
\hline Thyme & 0.985 \\
\hline Juniperus & 0.988 \\
\hline Oregano & 0.970 \\
\hline Cloves & 1.088 \\
\hline Cinnamon & 0.988 \\
\hline Tea tree & 1.026 \\
\hline Sunflower & 1.007 \\
\hline
\end{tabular}

No significant changes were observed between these intensity ratios, therefore we consider that all essential oils, including sunflower oil did not undergo cis-trans isomerization during the induced thermal oxidation.

\section{CONCLUSIONS}

The data obtained reflected the potential of ATR-FTIR-MIR method to obtain detailed information and specific patterns of different essential oils (thyme, oregano, juniper, clove, cinnamon, and tea tree). The different plantderived essential oils (from aromatic/medicinal and spices) showed specific FTIR spectra, and the qualitative features were identified in different zones, specific to molecular vibrations and related to their molecular composition. These data were in good agreement with our previous GC-MS analysis of the same samples [54].

Beside the qualitative patterns, semi-quantitative analysis was done, recording the peak intensities related to specific wavenumbers. Therefore, it was possible to realize specific "identity cards" of each oil, based on specific absorption wavenumbers and intensity range, to be markers of authenticity.

As a second goal, by an induced thermal oxidation it was demonstrated that almost all tested essential oils showed good oxidative stability. By routine chemical methods (peroxide index and malondialdehyde test) and evaluation of FTIR intensities at specific wavenumbers, there were find good, significant positive correlations. Therefore, specific FTIR wavenumbers from the fingerprint region can be considered as markers of oxidative stability of essential oils. 
All data obtained by ATR-FTIR-MIR spectroscopy were further processed by chemometrics, via multivariate analysis bringing added-value information and biological significance. By PLSDA-and Random Forest analysis significant correlations and sample discrimination were identified and finally reflected by intuitive heatmaps.

This study combined the capability of ATR-FTIR-MIR spectroscopy to realize a pattern recognition of essential oils as a rapid and non-destructive method, as well the oxidative modifications, easier than chemical routine tests. This study contributes to the control of quality, authenticity, and safety of essential oils and can be extended to other extracts, used as additives or ingredients in food or cosmetic products.

\section{EXPERIMENTAL SECTION}

\section{Materials}

A few types of essential oils (Thyme, Juniperus, Oregano, Clove, Cinnamon, Tee Tree) and one sunflower oil were subjected to FTIR analysis. These were provided by the Fares Company Orăştie and were previously characterized by various physical and chemical parameters mentioned in their Technical Specifications. The controlled thermo-oxidation was performed in a hot air oven at $180^{\circ} \mathrm{C}$ for 14 hours. The FTIR analysis was applied before and after oxidation to determine their specific pattern and to highlight the spectral changes induced by thermal oxidation.

\section{ATR-FTIR-MIR Spectroscopy}

The ATR-FTIR-MIR absorption spectra were recorded using the Shimadzu IR Prestige -21 spectrophotometer with horizontal ATR diamond accessory and a single PIKE reflection to hexane as background (control). The spectra were recorded on the wavelength range $3600-650 \mathrm{~cm}^{-1}$ at a resolution of $4 \mathrm{~cm}^{-1}$, and 64 scans for one spectrum. The raw data obtained were processed using IR Solution Software (Shimadzu) and OriginR 7SR1 Software (OriginLab Corporation, Northampton, USA).

According to literature data, the different absorptions were especially followed [37]. Table 4 include the most significant bands which correspond to specific vibrations of the molecules found in different EOs.

From each FTIR spectrum a matrix representing the peak intensities as a function of wavenumber (WN) was built and used for statistical analysis and data interpretation. 


\section{Relative density}

A pycnometer, before and after being filled with water at $20^{\circ} \mathrm{C}$ was weighed. The empty, dried pycnometer is filled with each sample (EO) brought to a temperature of $20^{\circ} \mathrm{C}$ and weighed. The difference represents the parameter $(\mathrm{m})$. The relative density of the sample is calculated according to the formula: $D=m / m_{1}$ where $D=$ relative density. Parameters $m$ and $m_{1}$ represent EOs and water weight, respectively expressed in $\mathrm{g}$.

\section{Refractive index}

A few drops from each EO were placed between the prisms of the refractometer. The boundary between the light and the dark area was brought to the intersection of the reticular wires. The value of the refractive index was read directly on the scale.

Table 4. Typical FTIR regions of volatile oils, correlated with the mode of vibration for specific functional groups, considered as predictor variables for statistical data processing.

\begin{tabular}{|c|c|c|}
\hline Range $\left(\mathrm{cm}^{-1}\right)$ & Functional Group & Mode of Vibration \\
\hline 3471 & $-\mathrm{C}=\mathrm{O}$ & Symmetric Stretching in esters \\
\hline 3006 & $-\mathrm{HC}=\mathrm{CH}-($ cis $)$ & Symmetric Stretching in cis olefins \\
\hline 2872,2962 & $=\mathrm{C}-\mathrm{H}$ (trans and cis) & Symmetric Stretching $\mathrm{C}-\mathrm{H}\left(\mathrm{CH}_{3}\right)$ \\
\hline 2854,2926 & $=\mathrm{C}-\mathrm{H}$ (trans and cis) & Symmetric Stretching $\mathrm{C}-\mathrm{H}\left(\mathrm{CH}_{2}\right)$ \\
\hline 1745 & $-\mathrm{C}=\mathrm{O}$ & Stretching \\
\hline $1635-1655$ & $\mathrm{RHC}=\mathrm{CH} 2$ & Stretching in cis olefins \\
\hline $1418,1458,1463$ & $=\mathrm{CH}_{2}$ & Deformation in plane \\
\hline $1376,1380,1396$ & $-\mathrm{CH}_{2-}$, dimethyl & $\begin{array}{c}\text { Plane \& out-of-plane deformations } \\
\text { in cis olefins }\end{array}$ \\
\hline $1147-1159$ & $-\mathrm{C}-\mathrm{O}, \mathrm{C}-\mathrm{OH}$ & Stretching, bending \\
\hline 1096,1237 & $-\mathrm{C}=\mathrm{O}$ & Stretching of ester groups \\
\hline 967 & $-\mathrm{HC}=\mathrm{CH}-($ trans $)$ & $\begin{array}{l}\text { Out of plane deformation for } \\
\text { disubstituted olefins }\end{array}$ \\
\hline $850-920$ & $=\mathrm{CH}_{2}$ & Out of plane deformation \\
\hline $842-862$ & Isopropyl group & Out of plane deformation \\
\hline 723,810 & $-\mathrm{C}-\mathrm{H} ;-\mathrm{HC}=\mathrm{CH}-($ cis $)$ & $\begin{array}{c}\text { Bending (out of plane) specific to } \\
\text { olefins }\end{array}$ \\
\hline
\end{tabular}

\section{Rotational power}

The rotational power was determined by a polarimeter, as follows: the tube with the polarimeter was filled with the sample, avoiding the formation of air bubbles. The angle of rotation alpha $( \pm)$ was read on the scale of the analyzer. 


\section{Peroxide Index}

Reagents: Chloroform: Acetic acid, 2:1, saturated potassium iodide solution, Natrium thiosulphate $0.01 \mathrm{~N}$, starch solution $1 \%$. To $15 \mathrm{ml}$ solvents mix there were added $2,5 \mathrm{~g}$ from each EO oil and fresh potassium iodide solution. After mixing $1 \mathrm{~min}, 30 \mathrm{ml}$ water was added and titrated with thiosulfate, using starch as color indicator. In parallel, a blank (distilled water) was titrated. The value of peroxide index (IP) was calculated according to the formula: $I P=10 x(V 2-V 1) / m$, where $m=2.5 \mathrm{~g}, \mathrm{~V} 2$ - thiosulfate volume used for samples titration; V1- thiosulfate volume used for blank.

\section{Lipoperoxidation}

Reactive oxygen species degrade polyunsaturated lipids to form malondialdehyde (MDA), which measure the degree of lipid peroxidation [57]. The spectrophotometric determination of MDA is done after its condensation with thiobarbituric acid (TBA) to give a fluorescent pink-red derivative (MDATBA complex) that can be measured by absorption at $535 \mathrm{~nm}$ [58]. A temperature of $100^{\circ} \mathrm{C}$ and a low $\mathrm{pH}$ (max. 3) are required to release MDA from oxidized lipids. To build the calibration curve for MDA, a solution $100 \mu \mathrm{M}$ $1,1,3,3$ tetramethoxypropane was used. Shortly, in a centrifuge tube, to 100 $\mu \mathrm{l}$ oil there were added $0.9 \mathrm{ml}$ Tris solution and $2 \mathrm{ml}$ of TBA reagent. After mixing 1 min and 15 min kept in a boiling water bath, the solution was centrifuged $10 \mathrm{~min}$ at $1000 \mathrm{rpm}$ and the pink MDA-TBA complex, was measured at $535 \mathrm{~nm}$ against a blank containing $1 \mathrm{ml}$ Tris and $2 \mathrm{ml}$ TBA reagent. The results (Eq MDA) were calculated by multiplying the Absorbance (A535) with the value of its absorption molar coefficient (163.8).

\section{Chemometrics}

The raw FTIR data representing the absorption intensities at each wavenumber from the middle infrared region $\left(3600-650 \mathrm{~cm}^{-1}\right)$ were statistically processed using the online software Metaboanalyst 5.0 (www.metaboanalyst.ca) and included the multivariate analysis by PLSDA (score plots and VIP value ranking), heatmaps, Random forest (RF) analysis.

\section{ACKNOWLEDGMENTS}

The technical support of the Spectroscopy laboratory - Institute of Life Sciences at University of Agricultural Sciences and Veterinary Medicine ClujNapoca is acknowledged. 


\section{REFERENCES}

1. M.D. Guillén; N. Cobo; J. Sci. Food Agric., 1997, 75, 1-11.

2. A.S. Franca; L.M.L. Nollet; Spectroscopic Methods in Food Analysis, CRC Press, NY, 2017, $664 \mathrm{pg}$.

3. D.-W. Sun; Infrared Spectroscopy for Food Quality Analysis and Control; Academic Press: Cambridge, MA, USA, 2009.

4. C. Socaciu; F. Ranga; F. Fetea; D. Leopold; F. Dulf; R. Parlog; Czech J. Food Sci., 2009, 27, S70-S75.

5. W.-H. Su; D.-W. Sun; Food Eng. Rev., 2019, 11, 142-158.

6. Q. Li; J. Chen; Z. Huyan; Y. Kou; L. Xu; X. Yu; J.-M. Gao; Crit. Rev. Food Sci. Nutr., 2019, 59, 3597-3611.

7. G. Gurdeniz; F. Tokatli; B. Ozen; Eur. J. Lipid Sci. Technol., 2007, 109, 1194-1202.

8. Y.W. Lai; E.K. Kemsley; R.H. Wilson; J. Agric. Food Chem., 1994, 42, 1154-1159.

9. D.A. Rusak; L.M. Brown; S.D. Martin; J. Chem. Educ., 2003, 80, 541-543.

10. L. Xu; X. Zhu; X. Yu; Z. Huyan; X. Wang; Eur. J. Lipid Sci. Technol., 2018, 120, 1700396.

11. J. Xia; N. Psychogios; N. Young; D.S. Wishart; Nucleic Acids Res., 2009, 37, W652-W660.

12. Y. Le Dreau; N. Dupuy; J. Artaud; D. Ollivier; J. Kister; Talanta, 2009, 77, 17481756.

13. L. Cerretani; A. Giuliani; R.M. Maggio; A. Bendini; T.G. Toschi; A. Cichell; Eur. J. Lipid Sci. Technol., 2010, 112, 1150-1157.

14. N.Vlachos; Y. Skopelitis; M. Psaroudaki; V. Konstantinidou; A. Chatzilazarou; E. Tegou; Anal. Chim. Acta, 2006, 459-465.

15. M.D. Guillen; N. Cabo; J. Sci. Food and Agr., 2000, 80, 2028-2036.

16. B. Muik; B. Lendl; A. Molina-Diaz; M. Valcarcel; M.J. Ayora-Canada; Anal. Chim. Acta, 2007, 593, 54-67.

17. M.A. Poiana; E. Alexa; M.F. Munteanu; C. Mateescu; Open Chemistry, 2015, 13, 689-698.

18. C. Socaciu; F. Fetea; F. Ranga; A. Bunea; F. Dulf; S. Socaci; A. Pintea; Appl. Sci. 2020, 10, 8695.

19. H. Yang; J. Irudayaraj; M.M. Paradkar; Food Chem., 2005, 93, 25-32.

20. L.E. Rodriguez-Saona; M.E. Allen Dorf; Ann. Rev. Food Sci. Technol., 2011, 2, 467-483.

21. A. Biancolillo; F. Marini; C. Ruckebusch; R. Vitale; Appl. Sci., 2020, 10, 6544.

22. A.C. Godoy; P.D.S. dos Santos; A.Y. Nakano; R.A. Bini; D.A.B. Siepmann; R. Schneider; P.A. Gaspar; P.W.D. Pfrimer; R.F. da Paz; O.O. Santos; Food Anal. Meth., 2020, 13, 1138-1147.

23. F. Bachion de Santana; W. Borges Neto; R.J. Poppi; Food Chem., 2019, 293, 323-332.

24. C. Fisher; T.R. Scott; Food Flavours: Biology and Chemistry, Royal Society of Chemistry, 2007, 176 pg, ISBN 9781847550866 
ATTENUATED TOTAL REFLECTANCE - FOURIER TRANSFORM INFRARED SPECTROSCOPY ...

25. J. Rivera Calo; P.G. Crandall, C.A. O'Bryan; S.C. Ricke; Food Control, 2015, 54, 111-119.

26. A. Wesołowska; D. Jadczak; Not. Bot. Horti. Agrobo., 2019, 47, 829-835.

27. V. Istudor; Farmacognozie, Fitochimie, Fitoterapie, vol. II, Ed. Medicală, Bucureşti, 2001

28. S.A. Johnson; Medicinal Essential Oils: The Science and Practice of EvidenceBased Essential Oil Therapy, Lightning Source Inc. Publ, 2017.

29. F. Bakkali; S. Averbeck; D. Averbeck; M. Idaomar; Food and Chem Toxicol., 2008, 46, 446-475.

30. S. Baptista-Silva; S. Borges; O.L. Ramos; M. Pintado; B. Sarmento; J. of Essential Oil Res., 2020, 32, 279-295.

31. T.K.T. Do; F. Hadji-Minaglou; S. Antoniotti; X. Fernandez; Trends Anal Chem., 2015, 66, 146-157.

32. I.L. Milovanović; A.C. Mišan; M.B. Sakač; I.S. Čabarkapa; B.M. Šarić; J.J. Matić; P.T. Jovanov; Food Process Quality Safety, 2009, 3-4, 75-79.

33. European Pharmacopoeia (Ph. Eur.) 10th Edition, 2020

34. O.Taylan; N. Cebi; O. Sagdic; Foods, 2021, 10, 202-212.

35. N. Cebi; O. Taylan; M. Abusurrah; O. Sagdic; Foods, 2021,10, 27-32.

36. J.D. Wicochea Rodriguez; S. Peyron; P.Rigou; P.Chalier; PLOS ONE, 2018,13, e0207401.

37. S. Agatonovic-Kustrin; P. Ristivojevic; V. Gegechkori; T.M. Litvinova; D.W. Morton; Appl. Sci., 2020, 10, 7294.

38. B. Bozin; N. Mimica-Dukic; N. Simin; G. Anackov; J Agric Food Chem., 2006, $54,1822-1828$.

39. C. Turek; F.C. Stintzing; Compr. Rev. Food Sci. Food Safety, 2013, 12, 40-53.

40. P. Satyal; B.L. Murray; R.L. Mcfeeters; W.N. Setzer; Foods, 2016, 5, 70-75.

41. A.K. Al-Asmari; T. Athar; A.A. Al-Faraidy; M.S. Almuhaiza; Asian Pac. J. Trop. Biomed., 2017, 7, 147-150.

42. J. Kula; T. Majda; A. Stoyanova; E. Georgiev; J. Essent. Oil Bear Plant., 2007, 10, 215-220.

43. X. Stoilova; S. Bail; G. Buchbauer; A. Krastanov; A. Stoyanova; E. Schmidt; et al ; Bosnia Nat. Prod. Commun., 2008, 3, 1043-1046.

44. Salamon; P. Petruska; Indian J.Pharmaceut.Educ. Res.,2017, 51, S479-S482.

45. S. Falcãoa; I. Bacémb; G. Igrejasb; P.J. Rodriguesc; M. Vilas-Boasa; J.S. Amarala; Ind Crops \& Prod., 2018,124, 878-884.

46. I. Amri; E. Mancini; L. De Martino; A. Marandino; H.Lamia; H. Mohsen; J. Bassem; M. Scognamiglio; E. Reverchon; V. De Feo; Int.J.Mol.Sci, 2012, 13, 16580-16591.

47. D. Gallart-Mateua; C.D. Largo-Arangoa; T. Larkmanb; S. Garriguesa; M. De La Guardia; Talanta, 2018, 189, 404-410.

48. K. Chaieb; H. Hajlaoui; T. Zmantar; A. Ben Kahla-Nakbi ; M, Rouabhia; K. Mahdouani; Phytotherapy Res. 2007, 21, 501-506.

49. H. Boughendjioua; IRJPMS, 2018, 11, 26-28.

50. G. Jayaprakasha; L. Rao; Crit Rev Food Sci Nutr. 2011, 51, 547-562. 
51. K.M. Brodowska; A.J. Brodowska; K. Śmigielski; E. Łodyga-Chruścińska; Eur J Biol Res., 2016, 6, 310-316.

52. J. Shao; Y. Zhang; Z. Zhu; Tropical J. Pharm. Res., 2018, 17, 1839-1845.

53. W. Zhou; Z. Liang; P. Li; Z. Zhao; J.Chen-Zhou; Chem. Central J., 2018, 12, 71-78.

54. R.M. Popa; S. Socaci; A. Farcas; C. Socaciu; Bull USAMV Food Sci Technol, 2021, 78, 88-100.

55. R.M. Popa; F. Fetea; C. Socaciu; Molecules, 2021, in press.

56. AOCS Official Method 14D-99, Rapid Determination of Isolated geometric trans Isomers in fats and oils by Attenuated Total Reflectance Infrared Spectrometry, $7^{\text {th }}$ edition, revised 2020.

57. M.W. Davey; E. Stals; B. Panis; J. Keulemans; R.L. Swennen; Anal. Biochem., 2005, 347, 201-207.

58. V. Nair; C.L. O'Neil; P.G. Wang; Malondialdehyde, Encyclopedia of Reagents for Organic Synthesis, John Wiley \& Sons, New York, 2008. 